\title{
Distinct activation of tumor necrosis factor- $\alpha$ and interleukin-6 in the spinal cord after surgical incision in rats
}

\author{
YAN-LING ZHANG ${ }^{1}$, JUN-MEI XU ${ }^{1}$, PEI ZHOU ${ }^{1}$, XIAO-LIN ZHONG ${ }^{2}$ and RU-PING DAI ${ }^{1}$ \\ ${ }^{1}$ Department of Anesthesia, The Second Xiangya Hospital of Central South University; ${ }^{2}$ Department of Anatomy, \\ Xiangya College of Medicine, Central South University, Changsha, Hunan, P.R. China
}

Received October 19, 2011; Accepted February 3, 2012

DOI: $10.3892 / \mathrm{mmr} .2012 .829$

\begin{abstract}
In a previous study, we showed that a deep thoracic incision induces the segmental upregulation of interleukin-1 $\beta$ (IL-1 $\beta$ ) in the spinal cord. However, whether the cytokines tumor necrosis factor- $\alpha$ (TNF- $\alpha$ ) and interleukin-6 (IL-6) are also activated in response to surgical incision remains to be determined. The present study aimed to investigate the expression pattern of TNF- $\alpha$ and IL- 6 in the spinal cord following a deep thoracic incision. After surgical incision, the mRNA levels of TNF- $\alpha$ and IL-6 in the thoracic spinal cord were transiently upregulated as determined by real-time polymerase chain reaction (PCR) assay. However, the activation of IL- 6 was detected at $1 \mathrm{~h}$ postoperatively, which was earlier compared to that of TNF- $\alpha$, observed at $6 \mathrm{~h}$ postoperatively. The activated TNF- $\alpha$ was mainly localized in the neurons, but not in microglia or astrocytes as determined by immunohistochemistry and confocal microscopy. However, the increased IL-6-immunoreactivity was mainly expressed in blood vessels. The differential upregulation of TNF- $\alpha$ and IL- 6 induced by incision suggests that the proinflammatory cytokines may play different roles in the development of surgical pain.
\end{abstract}

\section{Introduction}

Cytokines are highly potent, pleiotrophic and bioactive endogenous peptides produced by almost every nucleated cell and they are involved in a variety of physiological and pathological processes (1). In the nervous system, cytokines, released from glial cells and neurons under certain conditions, have various roles such as engaging in NMDA receptor-mediated neurotoxicity (2) and acute neurodegeneration (3). Results of previous studies showed that proinflammatory cytokines, such as tumor necrosis factor- $\alpha$ (TNF- $\alpha)$, interleukin-1 $\beta$ (IL-1 $\beta)$ and inter-

Correspondence to: Dr Ru-Ping Dai, Department of Anesthesia, The Second Xiangya Hospital of Central South University, Changsha, Hunan 410011, P.R. China

E-mail: ruping_dai@yahoo.com.cn

Key words: incisional pain, spinal cord, cytokine, tumor necrosis factor- $\alpha$, interleukin- 6 , interleukin 1- $\beta$ leukin-6 (IL-6), are strongly associated with pathological pain states and likely to induce the development of hyperalgesia at the level of spinal cord (4). Acute peripheral inflammation has been shown to induce the activation of astrocytes as well as the expression of IL-1 $\beta$ in the spinal cord, which correlate with zymosan- and formalin-induced allodynia (5). Additionally, the increase in TNF- $\alpha$ expression in the spinal cord correlates with the development of peripheral nerve injury and the endoneurial injection of TNF- $\alpha$ induces neuropathic pain in rats $(6,7)$. In a previous study, we showed that surgical incision results in the glial activation and segmental upregulation of IL-1 $\beta$ in the spinal cord (8). Notably, the increased IL-1 $\beta$ expression is mainly localized in the neurons but not in the glial cells (8). These findings suggest that the neuronal IL-1 $\beta$ activation may be involved in the pain induced by incision, a hallmark of postoperative pain. However, whether the cytokines TNF- $\alpha$ and IL- 6 are also activated in the spinal cord after surgical incision remains to be determined.

The present study aimed to examine the expression pattern of TNF- $\alpha$ and IL- 6 after surgical incision. Findings showed that surgical incision resulted in distinct expression profiles of TNF- $\alpha$ and IL- 6 following a deep thoracic incision. The distinct activation of TNF- $\alpha$ and IL- 6 may have different roles in the development of surgical pain.

\section{Materials and methods}

Animals. Experiments were performed on adult male TokyoWistar rats housed under standard environmental conditions and maintained on commercial rat chow and tap water ad libitum. The experimental protocol was approved by the Animal Care and Use Committee of Central South University and conformed to the National Institutes of Health Guide for the Care and Use of Laboratory Animals.

Surgical preparation and experimental protocol. An incisional animal model was established through a deep thoracic incision, as previously described by our team (8). In brief, adult male rats weighing 250-300 g, and in general good health were anesthetized with isoflurane. Under sterile conditions, a skin incision of approximately $1 \mathrm{~cm}$ was made in the midline of the thorax, starting from the sternal angle and running parallel to the sternum. The incision wound was deepened until the sternum was exposed, but remained intact. The thoracic 
Table I. Primers of the real-time polymerase chain reaction (PCR) assay.

\begin{tabular}{lllc}
\hline & \multicolumn{1}{c}{ Sense (5'-3') } & Anti-sense (3'-5') & Size (bp) \\
\hline IL-6 & gggactgatgttgttgacag & tgttcttcacaaactccagg & 304 \\
TNF- $\alpha$ & cccagaccctcacactcagat & ttgtccttgaagagaacctg & 215 \\
GAPDH & accaccatggagaaggctgg & ctcagtgtagcccaggatgc & 528 \\
\hline
\end{tabular}

IL-6, interleukin-6; TNF- $\alpha$, tumor necrosis factor- $\alpha$; GAPDH, glyceraldehyde-3-phosphate dehydrogenase.

musculature and skin incision were then closed by 3-0 suture. The sham-operated animals underwent the same procedure except that the incision was not carried out.

For RNA extraction, the experimental rats following deep thoracic incision were randomly divided and sacrificed at 1 and $6 \mathrm{~h}$, and on days 1 and 3 ( $\mathrm{n}=4$ at each group). Age-matched, sham-operated rats were used as the controls. The first and second segments of the thoracic spinal cord (T1 and T2) were collected at different time intervals and frozen in liquid nitrogen. The segments were maintained in a $-80^{\circ} \mathrm{C}$ freezer until required. In a separate setup, the sham-operated and various groups of experimental rats $(n=3)$ were perfused using $2 \%$ paraformaldehyde for immunohistochemistry.

RNA extraction and reverse transcription. Total RNA was isolated from the tissue using TRIzol ${ }^{\circledR}$ reagent based on the company protocol (Invitrogen, Grand Island, NY, USA). The RNA concentration was determined using spectrophotometry at $260 \mathrm{~nm}$. RNA integrity was electrophoretically verified. For the reverse transcription, the reaction mixture containing $2 \mu \mathrm{g}$ of RNA, $2.5 \mu \mathrm{m}$ of oligo(dT) primer, and 5 units of Molony murine leukemia virus reverse transcriptase (M-MLV, Promega, Madison, WI, USA) in a total volume of $25 \mu 1$, was incubated for $1 \mathrm{~h}$ at $42^{\circ} \mathrm{C}$ and stopped by heating for $5 \mathrm{~min}$ at $95^{\circ} \mathrm{C}$. The cDNA was quantified using spectrophotometry and diluted to $10-50 \mu \mathrm{g} / \mu \mathrm{l}$ for polymerase chain reaction (PCR) or real-time PCR assay.

Real-time PCR assay. PCR amplification efficiency analysis was performed using the LightCycler instrument (Roche, Germany). SYBR-Green I, a DNA binding dye, which binds in the minor groove of double-stranded DNA, was used to perform the PCR amplification assay. Q-Gene (9), the Microsoft ${ }^{\circledR}$ Excel ${ }^{\circledR}$-based software coded in Visual Basic was used to calculate the gene expression in the present study. In brief, PCR amplification conditions for the specific gene were optimized for the LightCycler instrument. cDNAs were amplified in the reaction mixture $(20 \mu \mathrm{l})$ containing LightCycler ${ }^{\mathrm{TM}}$-FastStart DNA Master SYBR-Green I, $0.5 \mu \mathrm{m}$ of each primer, and appropriate concentrations of $\mathrm{MgCl}_{2}$ using LightCycler. The level of specific gene expression was quantified and expressed as $\mathrm{C}_{t}$, the cycle number at which the LightCycler System detected the upstroke of the exponential phase of PCR product accumulation. After 40 cycles of PCR, the amplified product showed a single prominent band in the expected size for the specific gene. The primers corresponding to the rat TNF- $\alpha$, IL-6, and the reference gene, glyceraldehyde-3-phosphate dehydrogenase (GAPDH) are listed in Table I. The samples were diluted to appropriate concentrations to achieve the same efficiency for the specific gene and performed in triplicate. The specific genes were normalized to the level of GAPDH expression in each individual sample when all the data analyzed using the software mentioned above. The mean normalized expression was further performed for statistical analysis.

Immunohistochemistry and double labeling immunofluorescence. $\mathrm{T} 1$ and $\mathrm{T} 2$ segments of the spinal cord from the sham-operated and experimental rats were fixed for $4 \mathrm{~h}$ with $2 \%$ paraformaldehyde following perfusion and cryoprotected by immersion in $20 \%$ sucrose in phosphate buffer ( $\mathrm{pH} 7.4$ ). Transverse sections of the spinal cord were cut at $30 \mu \mathrm{m}$ with a cryostat and mounted on 3-aminopropyl triethoxy-silanecoated slides. Rabbit anti-TNF- $\alpha$ (dilution 1:200; Chemicon, Rosemont, IL, USA), rabbit anti-IL-6 (dilution 1:200; Santa Cruz Biotechnology, Santa Cruz, CA, USA) were incubated at room temperature overnight. The secondary reagents used for cytokine localizations were biotinylated goat anti-rabbit IgG or horse anti-mouse and $\mathrm{ABC}$ kit (Vector Laboratories, Burlingame, CA, USA). The peroxidase reaction was visualized using 3,3'-diaminobenzidine tetrahydrochloride (DAB, Sigma, St. Louis, MO, USA) as a peroxidase substrate.

Cells expressing IL- 6 or TNF- $\alpha$ were identified using double labeling. The first primary antibodies used were either polyclonal rabbit anti-IL-6 or anti-TNF- $\alpha$ (dilutions 1:100). The second primary antibodies were either mouse anti-OX-42 (dilution 1:2000), anti-GFAP (dilution 1:2000), anti-NeuN (dilution 1:1000, Chemicon) or anti-CD-31 [CD-31 is a major component of the endothelial intracellular junction (10)] (Dako, Glostrup, Denmark) were applied to the same sections simultaneously. Following incubation, sections were placed in $5 \mu \mathrm{g} / \mathrm{ml}$ Alexa Fluor 488 goat anti-mouse and 594 goat antirabbit $\operatorname{IgG}_{1}$ conjugate (Molecular Probes, Eugene, OR, USA) for $1 \mathrm{~h}$ in the dark at room temperature. After further washes, the preparations were mounted in Vectashield mounting medium (Vector Laboratories). Negative controls were performed routinely by incubating the sections in a buffer or normal-buffered serum instead of the primary antibody.

Statistical analysis. Statistical analysis was performed using software SPSS 10.0 (SPSS Inc). The data were presented as the mean \pm SEM. To evaluate the statistical significance, one-way ANOVA followed by Tukey test were performed with multiple-comparison procedure. When appropriate, unpaired t-tests were used to compare the experimental and sham groups. $\mathrm{P}<0.05$ was considered to indicate a statistically significant difference. 
A

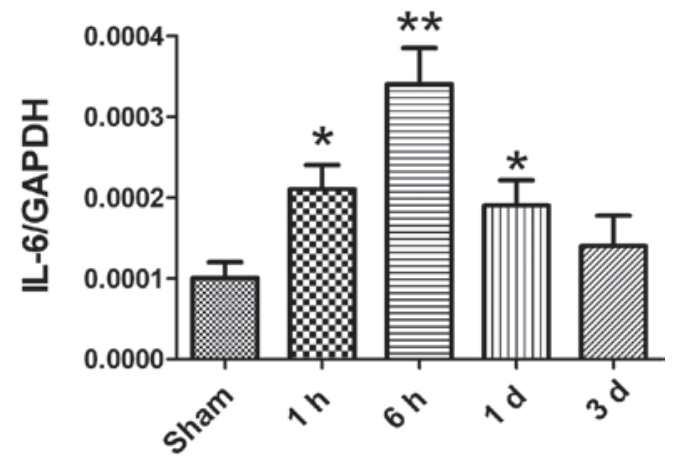

B

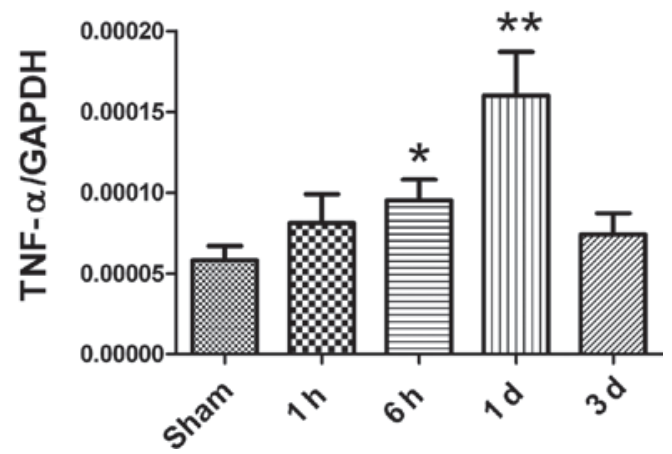

Figure 1. Relative expression of mRNA levels of (A) interleukin-6 (IL-6) and (B) tumor necrosis factor- $\alpha$ (TNF- $\alpha$ ) in different groups following incision and sham-operated rats is shown. (A) A transient increase of IL-6 mRNA levels in the thoracic segments of the spinal cord is evident 1 and $6 \mathrm{~h}$ after the operation. (B) TNF- $\alpha$ mRNA levels are upregulated, compared with those of sham-operated rats, $1 \mathrm{~h}$ after the operation, reaching peak level 1 day following the incision. Values are the mean \pm SEM $(n=4$ for the experimental and control groups, respectively).

\section{Results}

Real-time PCR assay showed that the gene expression of IL-6 and TNF- $\alpha$ in the T1 and T2 segments was transiently increased in response to a deep thoracic incision. However, the time intervals of the gene expression was different. The increase in IL-6 mRNA levels was observed at $1 \mathrm{~h}$ and reached the highest level at $6 \mathrm{~h}$ postoperatively (Fig. 1A). However, the upregulation of TNF- $\alpha$ mRNA levels was detected at $6 \mathrm{~h}$ and peaked 1 day postoperatively (Fig. 1B). Three days after the operation, it was noted that the gene expression of the two cytokines was not significantly different compared to that of the control.

The staining of TNF- $\alpha$ was also increased in response to the incision. The staining for TNF- $\alpha$ was extremely weak in the dorsal (Fig. 2A) and ventral horns (Fig. 2B) of the spinal cord in the sham-operated rats. TNF- $\alpha$-immunoreactivity (IR) was clearly increased at $6 \mathrm{~h}$ (Fig. 2C and D) and was the most intensive 1 day after the operation (Fig. 2E and F). The TNF- $\alpha$-IR cells were mainly localized in the dorsal and ventral horns and some of these cells were also positive for NeuN (Fig. 3G-I). Few co-localizations between TNF- $\alpha$ and either OX-42 (Fig. 3A-C) or GFAP (Fig. 3D-F) were observed in the spinal cord following the operation. This observation suggests that TNF- $\alpha$ is mainly expressed in the neurons in the $\mathrm{T} 1$ and $\mathrm{T} 2$ segments of the spinal cord after the incision.

The IL- 6 positive staining was relatively mild in the shamoperated rats (Fig. 4A). The obvious upregulation of IL-6-IR was evident in the T1 and T2 segments (Fig. 4B). IL-6 positive

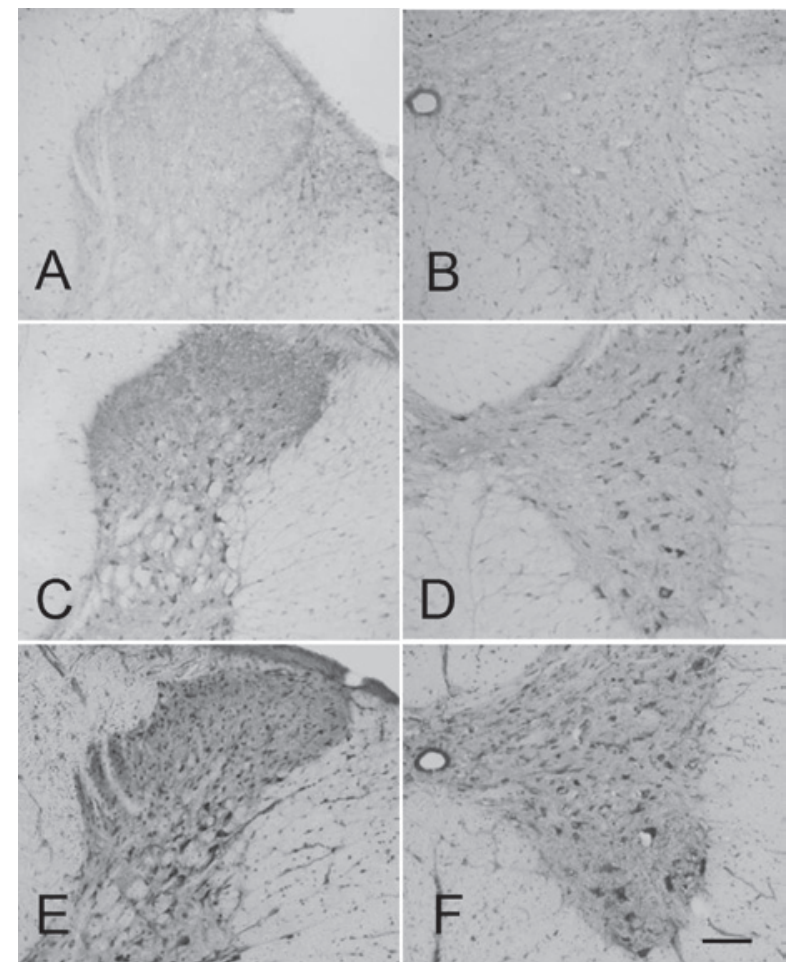

Figure 2. Tumor necrosis factor- $\alpha(\mathrm{TNF}-\alpha)$-immunoreactivity (IR) is evident in the dorsal and ventral horns of the T1 and T2 segments following the operation. (A) Weak staining is detected in the dorsal and (B) ventral horns in the sham-operated rats. (C and D) The staining increases in the dorsal and ventral horns in rats $6 \mathrm{~h}$ after the operation. (E and F) One day after the operation, some TNF- $\alpha$-immunoreactive cells appear to be intensely stained in neurons in the spinal cord observed under the microscope. Scale bars, $50 \mu \mathrm{m}$.

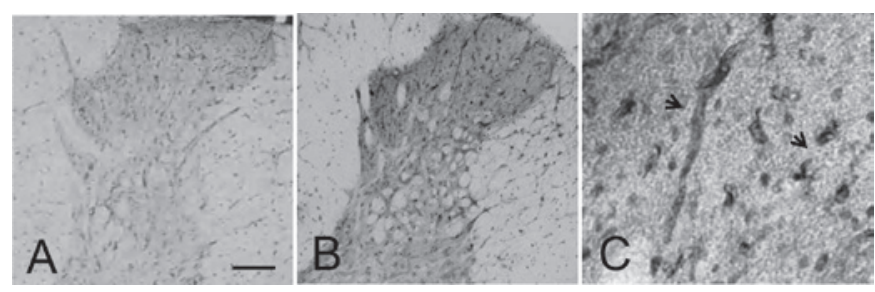

Figure 4. Representative images of interleukin-6(IL-6)-immunoreactivity (IR) in the spinal cord following a deep thoracic incision. (A) Weak staining of IL-6 in the dorsal horn of the thoracic spinal cord in the sham-operated rats is evident. (B) Increased IR to IL-6 is detected in the thoracic spinal cord in the rats $6 \mathrm{~h}$ after the operation. (C) IL-6-IR cells are mainly localized inthe blood vessels (arrows). Scale bars, (A and B) $100 \mu \mathrm{m}$, respectively, and (C) $25 \mu \mathrm{m}$.

staining appeared to be localized in the blood vessels (Fig. 4C). Double labeling immunofluorescence showed that there was no co-localization either between OX-42-IR and IL-6-IR cells (Fig. 5A-C), or between GFAP-IR and IL-6-IR cells in the spinal cord. Some IL-6-IR cells were also immunoreactive for NeuN, indicating that IL-6 was able to be released from the neurons (Fig. 5D and E). Numerous IL-6 labeling cells were expressed in the CD-31 labeling cells (Fig. 5G-I).

\section{Discussion}

Extensive studies suggest that proinflammatory cytokines, in particular, TNF- $\alpha$, IL-1 $\beta$ and IL- 6 , are involved in the pathological pain states including hyperalgesia and allodynia (4). 


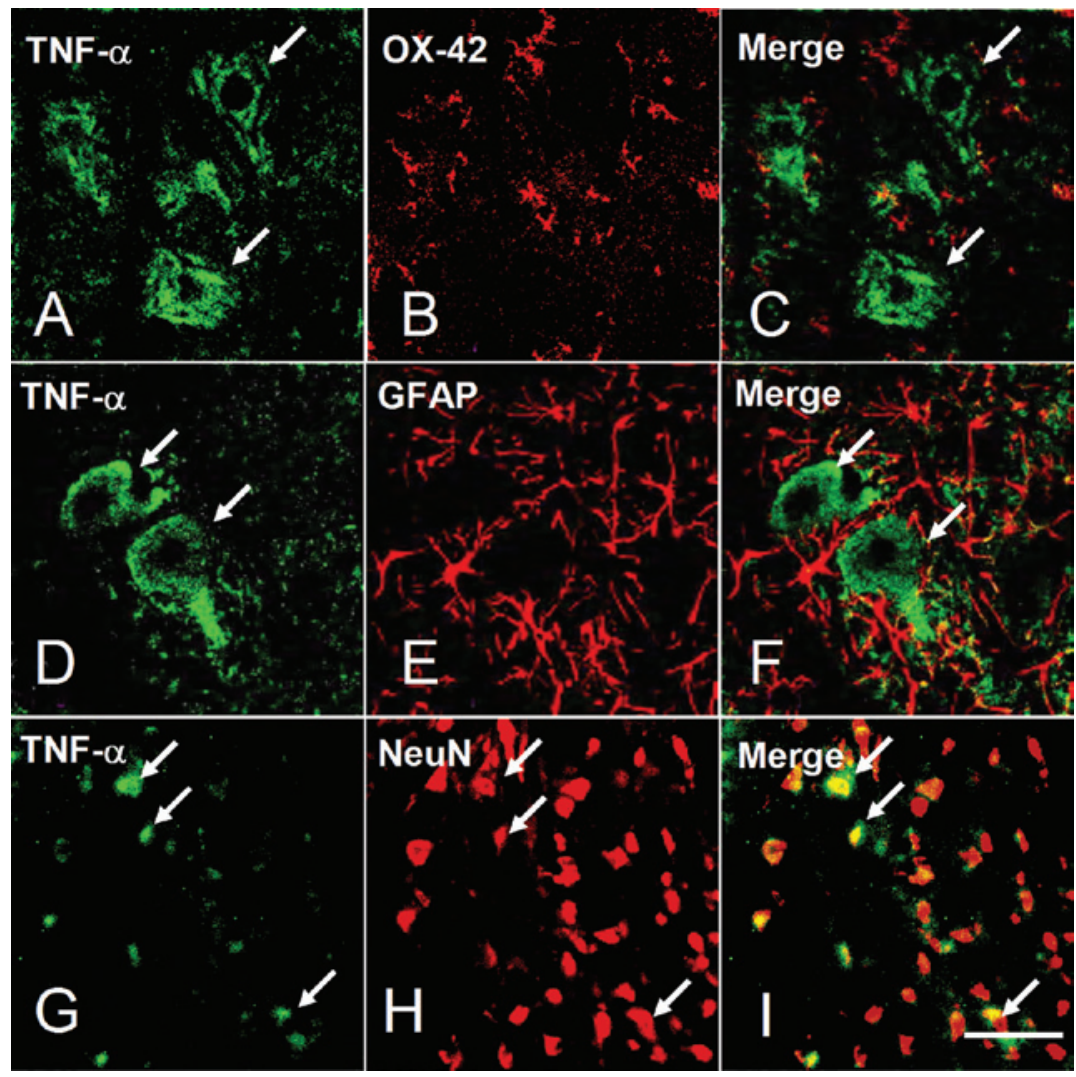

Figure 3. Double labeling immunofluorescence between (A-C) tumor necrosis factor- $\alpha$ (TNF- $\alpha$ ) (green) and OX-42 (red), (D-F) between TNF- $\alpha$ (green) and GFAP (red), and (G-I) between TNF- $\alpha$ and NeuN (red) in the thoracic spinal cord is evident $6 \mathrm{~h}$ after the operation. Some TNF- $\alpha$-immunoreactive (IR) cells are also stained for NeuN (arrows). Scale bars, $25 \mu \mathrm{m}$.

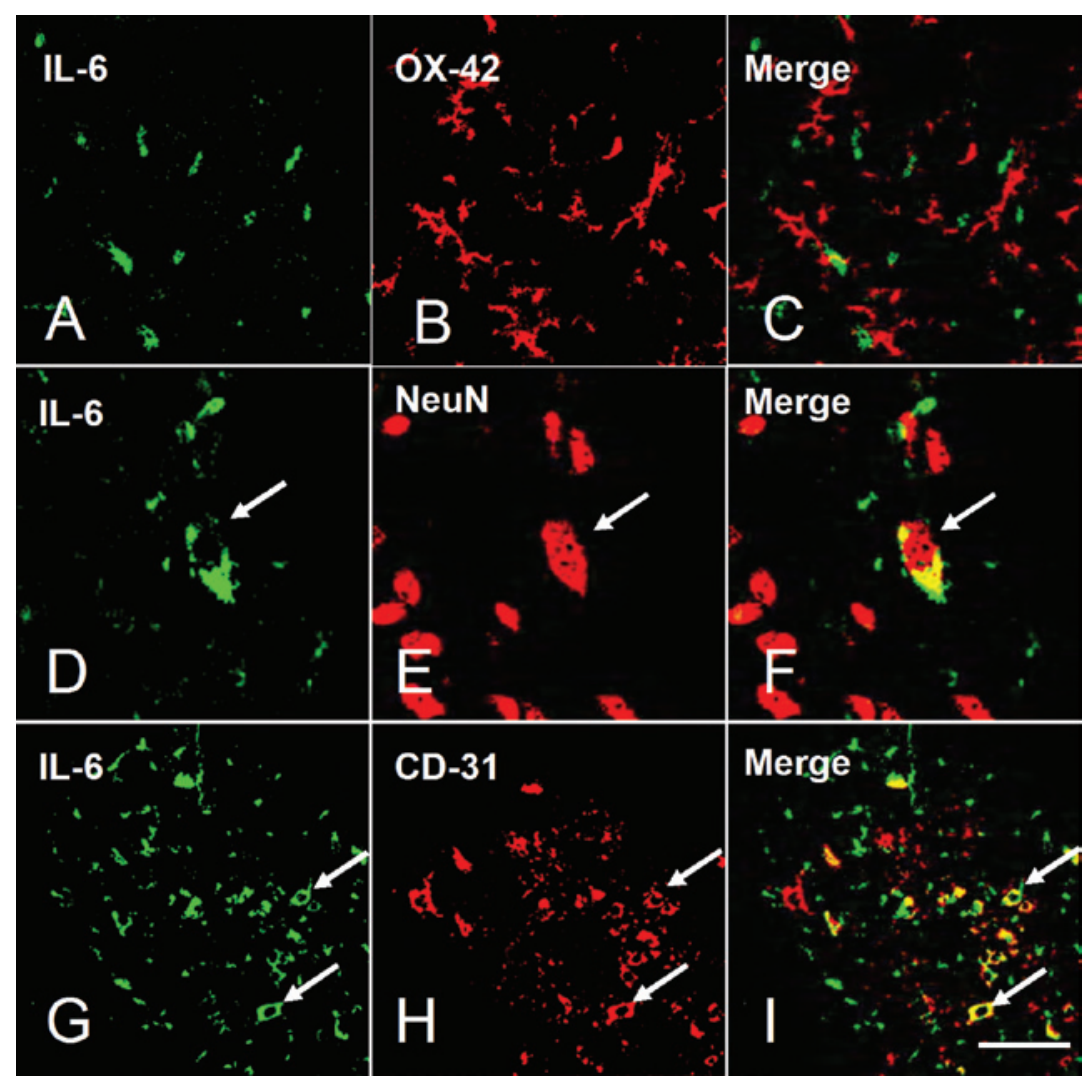

Figure 5. (A-C) Double labeling immunofluorescence between interleukin-6 (IL-6) (green) and OX-42 (red), (D-F) between IL-6 (green) and NeuN (red), and (G-H) between IL-6 (green) and CD-31 (red), is evident in the T1 and T2 segments of the spinal cord in rats $6 \mathrm{~h}$ following the incision. (D-F) Co-localization (arrows) is detected between NeuN and IL-6. Few co-localizations between GFAP and IL-6 are evident. (G-H) Abundant IL-6-IR cells are localized with CD-31 positive staining (arrows) in the spinal cord. Scale bars, $25 \mu \mathrm{m}$. 
In addition, it is believed that TNF- $\alpha$, IL-1 $\beta$ and IL- 6 are capable of acting on cells including neurons and glial cells with their coordinated production and action, known as 'cytokine cascade' or 'cytokine network' $(11,12)$. TNF- $\alpha$, which is known to be an upstream cytokine of the cytokine network, induced the activation of IL-1 $\beta$. Moreover, both TNF- $\alpha$ and IL-1 $\beta$ were able to activate IL-6, whereas IL-6 was not able to activate IL-1 $\beta$ or TNF- $\alpha$ (13). Findings of our previous study have demonstrated the segmental upregulation of IL-1 $\beta$ in response to surgical incision (8). In the present study, TNF- $\alpha$ was activated and mainly expressed in the neurons in the thoracic spinal cord following incision. The similarity in the tempo-spatial upregulation of TNF- $\alpha$ after surgical incision suggest that a correlation exists between TNF- $\alpha$ and IL-1 $\beta$ in the development of surgical pain. In the present study, IL-6 mRNA and protein levels were also activated after surgical incision. However, unlike TNF- $\alpha$ and IL-1 $\beta$, which were mainly expressed in the neurons, the increased level of IL-6 was mainly expressed in the blood vessels. Although TNF- $\alpha$ and IL-1 $\beta$ were capable of inducing the activation of IL- 6 under various conditions, the distinct cell locations of IL-6 from TNF- $\alpha$ and IL- $1 \beta$ suggest that IL- 6 may have different roles in postoperative pain.

Previous results have shown that astrocytes and microglial cells became activated in response to hindpaw incision (8). Although glial cells are believed to release the proinflammatory cytokines in most circumstances, few co-localizations were detected between glial cells and the measured cytokines in the present study. This observation suggests that these cells may not be responsible for the production of the proinflammatory cytokines in incisional pain. TNF- $\alpha$ and IL-1 $\beta$ have been observed mainly in neurons, whereas IL- 6 was present in the blood vessels as well as neurons in the T1 and T2 segments of the spinal cord. The expression of IL- 6 within the blood vessels suggests that the production of IL-6 may be, partially at least, a result of the inflammatory mediators released from the injured tissue. The upregulation of IL-6 within blood vessels, in turn, may be involved in central sensitization via the activation of cyclooxygenase-2 (COX-2) and prostaglandin E2, well-known mediators contributing to various pathological pain states in the blood-nerve barrier $(1,14,15)$.

Another observation of the present study is the upregulation of TNF- $\alpha$ and IL- 6 in dorsal and ventral horns in the thoracic segments of the spinal cord. The reason for the upregulation of TNF- $\alpha$ and IL- 6 in the ventral horn remains unknown. The two cytokines were mainly localized in the dorsal horn where the neurons sensitize the nociceptive inputs from the periphery in response to a peripheral tissue injury. After the injury, multiple chemical mediators, released from damage and inflammatory cells, activated nociceptors or acted together to produce sensitization in the somatosensory nervous system $(16,17)$. However, in spite of the unclear mechanism, the upregulation of TNF- $\alpha$ and IL-6 in the ventral horn suggests that their involvement may not be limited to the contribution of pathological pain states.
In conclusion, in the present study, TNF- $\alpha$ and IL-6 were activated in the thoracic spinal cord in response to the deep thoracic incision. However, the activated TNF- $\alpha$ was mainly expressed in the neurons, whereas IL-6 was mainly localized in the blood vessels. The distinct expression of TNF- $\alpha$ and IL-6 suggests that these cytokines may not be associated with postoperative pain.

\section{Acknowledgements}

This study is supported by the National Natural Science Foundation of China (NSFC 81070897) to Dr R. Dai.

\section{References}

1. Samad T, Moore K, Sapirstein A, Billet S, Allchorne A, Bonventre JV and Woolf CJ: Interleukin-1beta-mediated induction of Cox-2 in the CNS contributes to inflammatory pain hypersensitivity. Nature 410: 471-475, 2001.

2. Ma X, Gottschall P, Chen L, Wiranowska M and Phelps C: Role and mechanisms of interleukin-1 in the modulation of neurotoxicity. Neuroimmunomodulation 10: 199-207, 2002.

3. Allan S and Rothwell N: Cytokines and acute neurodegeneration. Nat Rev Neurosci 2: 734-744, 2001.

4. DeLeo J and Yezierski R: The role of neuroinflammation and neuroimmune activation in persistent pain. Pain 90: 1-6, 2001.

5. Bao L, Zhu Y, Elhassan A, Wu Q, Xiao B, Zhu J and Lindgren J: Adjuvant-induced arthritis: IL-1 beta, IL-6 and TNF-alpha are upregulated in the spinal cord. Neuroreport 12: 3905-3908, 2001.

6. Wagner R and Myers R: Endoneurial injection of TNF-alpha produces neuropathic pain behaviors. Neuroreport 7: 2897-2901, 1996.

7. Wagner R and Myers R: Schwann cells produce tumor necrosis factor alpha: expression in injured and non-injured nerves. Neuroscience 73: 625-629, 1996.

8. Fu D, Guo Q, Ai Y, Cai H, Yan J and Dai R: Glial activation and segmental upregulation of interleukin-1beta (IL-1beta) in the rat spinal cord after surgical incision. Neurochem Res 31: 333-340, 2006.

9. Muller PY, Janovjak H, Miserez AR and Dobbie Z: Processing of gene expression data generated by quantitative real-time RT-PCR. Biotechniques 32: 1372-1379, 2002.

10. van Mourik JA, Leeksma OC, Reinders JH, De Groot PG and Zandbergen-Spaargaren J: Vascular endothelial cells synthesize a plasma membrane protein indistinguishable from the platelet membrane glycoprotein IIa. J Biol Chem 260: 11300-11306, 1985.

11. Blackwell $\mathrm{T}$ and Christman $\mathrm{J}$ : The role of nuclear factor-kappa $\mathrm{B}$ in cytokine gene regulation. Am J Respir Cell Mol Biol 17: 3-9, 1997.

12. Mann D: Inflammatory mediators and the failing heart: past, present, and the foreseeable future. Circ Res 91: 988-998, 2002.

13. Watkins L and Maier S: The pain of being sick: implications of immune-to-brain communication for understanding pain. Annu Rev Psychol 51: 29-57, 2000.

14. Cartmell T, Poole S, Turnbull A, Rothwell N and Luheshi G: Circulating interleukin- 6 mediates the febrile response to localised inflammation in rats. J Physiol 526: 653-661, 2000.

15. Ek M, Engblom D, Saha S, Blomqvist A, Jakobsson PJ and Ericsson-Dahlstrand A: Inflammatory response: pathway across the blood-brain barrier. Nature 410: 430-431, 2001.

16. Sweitzer S, Colburn R, Rutkowski M and DeLeo J: Acute peripheral inflammation induces moderate glial activation and spinal IL-1beta expression that correlates with pain behavior in the rat. Brain Res 829: 209-221, 1999.

17. Boddeke EW: Involvement of chemokines in pain. Eur J Pharmacol 429: 115-119, 2001. 